Original Research Paper

\title{
Crosstalk between Oxidative Stress Signaling and HER2 Pathway in Breast Cancer
}

\author{
${ }^{1}$ Vanessa Jacob Victorino, ${ }^{2}$ Adriano Martin Felis Aranome, ${ }^{2}$ Fernanda Carolina de Campos, ${ }^{2}$ Ana Cristina \\ da Silva do Amaral Herrera, ${ }^{2}$ Rubens Cecchini and ${ }^{2,3}$ Carolina Panis \\ ${ }^{1}$ Laboratory of Medical Investigation (LIM51), Department of Emergency Medicine, School of Medicine, University of São \\ Paulo, São Paulo, Brazil \\ ${ }^{2}$ Laboratory of Pathophysiology and Free Radicals, Department of General Pathology, State University of Londrina, Londrina, \\ Paraná, Brazil \\ ${ }^{3}$ Laboratory of Inflammatory Mediators, State University of West Paraná, UNIOESTE, Francisco Beltrão, Brazil
}

Article history

Received: 05-02-2014

Revised: $14-02-2014$

Accepted: 05-01-2015

Corresponding Author: Vanessa Jacob Victorino Laboratory of Medical Investigation (LIM51), Department of Emergency Medicine, School of Medicine, University of São Paulo, São Paulo, Brazil Email: carolpanis@sercomtel.com.br

\section{Introduction}

Breast cancer is the most lethal malignancy in women worldwide, with the highest incidence in both developed and developing countries (WHO, 2014). The development of breast cancer is multifactorial, where environmental and genetic susceptibility may play an important role (Johnson-Thompson and Guthrie, 2000).

Tumors are clinically characterized according to the TNM classification ( $\mathrm{T}$, tumor; $\mathrm{N}$, node; and $\mathrm{M}$, metastasis). The staging of breast cancer, proposed by the International Union Against Cancer (UICC), comprises the tumor size, presence/absence of metastases in lymph nodes and presence/absence of distant metastases (Barros et al., 2001; INC, 2004).

Staging of tumors according to TNM classification allows the tumor characterization, but it failures to therapeutic indication (Vieira et al., 2008). Perou et al. (2000) characterized the variation of gene expression patterns in breast tumor samples using DNA microarray. T0he patterns provided a distinct molecular portrait of each tumor, whose standards reflect a relationship between tumors and connections between a specific gene and specific tumor. According to this classification, tumors were identified into four different groups: Luminal A and B (positive tumors expressing estrogen receptors-ER and progesterone receptors-PR), triple negative (also called basal-like) and HER2overexpressed (human epidermal growth factor 2, also known as ErbB2) (Sioshansi et al., 2011).

Therefore, therapeutic targets have being formulated aiming at the tumor molecular subtype. During the development of breast cancer, about $20 \%$ of patients are positive for HER2 amplification or overexpression. HER2-overexpression may be due to gene amplification or transcriptional deregulation (Gutierrez and Schiff, 2011) and it has been implicated as important target for therapies, even in tumors that have concomitant ER and PR (Dogan et al., 2011; Hynes and Lane, 2005). However, several women may become resistant to therapy (Nahta et al., 2006).

\section{HER2 Signaling Pathways}

HER2 receptors are included in the subclass I receptor tyrosine kinase, which comprises four members: 
HER1, HER2, HER3 and HER4. Through distinct signaling pathways, ligand-dependent HER family receptors mediate several transcription factors that act in the control of apoptosis, migration, growth, adhesion and cell differentiation. During physiological processes, HER-mediated pathways are related to embryogenesis, organogenesis, tissue regeneration and wound healing (Yarden and Sliwkowski, 2001).

In the cell membrane, the HER2 receptor is found as homodimer or heterodimer (Fig. 1). The HER2 dimmers have prolonged activity and attenuate signaling evasion (Yarden, 2001). HER family receptors have an extracellular region composed of four areas: I-IV. The HER2 extracellular conformation is fixed and resembles a state of "ligand-activated", as the II-IV interaction domains are absent and the dimerization loop in II domain is exposed. This conformational state makes HER2 as a preferred dimerization partner for others HER family members. Also, the interaction of HER2 with a ligand is not possible due to its inaccessible binding site (Hynes and Lane, 2005).

Signaling pathways activated by ligands to HER2/HER 1 heterodimer act directly in Phospholipase C $\gamma 1$ (PLC $\gamma 1$ ) through the phosphorylated tyrosine residue 1248 of HER2 receptor. This pathway has indirect relationship with Protein Kinase C (PKC) pathway. Signals originated by this pathway oscillate the intracellular calcium concentration and the reorganization of actin filaments, leading to projections formation of the cytoskeleton. Thus, the activation of PLC pathway is directly related to increased migration and tumor progression (Dittmar et al., 2002). Besides, the activation of the HER2/ HER1 heterodimer may promote tumor progression and proliferation. HER2overexpression activates this pathway and amplifies the Ras signaling complex through Growth factor receptorbound protein 2/Son Of Sevenless (Grb2/SOS), thus, acting on nuclear cyclin D1, Cyclin Dependent kinase K6 (CDK6), cyclin E and p27KIP1 (Janes et al., 1994).

Once HER2 receptor is presented as homodimer in the cell membrane, there is no known ligand (Yarden and Sliwkowski, 2001). As homodimer, HER2 acts on the PAR complex, decoupling PAR-3 of the active complex PAR6-PAR3-aPKC-CDC42 and binding aPKC to PAR6. The PAR complex dissociation disrupts the normal organization of the epithelium, leading to uncontrolled proliferation and protection from apoptosis (Aranda et al., 2006).

It is well described that intracellular signaling pathways mediated by HER2 also include ras-Mitogen Activated Protein Kinase (ras-MAPK), S6 kinase independent MAPK and phospholipase C-gamma pathways. Nevertheless, the biological consequences of these pathways activation are not completely understood
(Kurebayashi, 2001). Tanizaki et al. (2011) reported that HER2-overexpression can activate Mitogen-activated protein kinase/Extracellular signal-regulated KinaseExtracellular signal-Regulated Kinase (MEK-ERK) signaling pathways as a mechanism to inhibit apoptosis.

The HER2 dimer is the preferred dimerization partner for all HER family members (Graus-Porta et al., 1997). Zhou and Agazie (2011) demonstrated that overexpression of HER2 itself increases basal signaling cascade stimulated per se. However, when stimulated by Epidermal Growth Factor (EGF), even at low concentrations, an increase of the intensity of signals is observed. It was also demonstrated by those authors that when stimulation of EGFR is inhibited, suppression of EGFR-ERK1/2-AKT (protein kinase B) pathway is observed in the presence of HER2overexpression. Thus, it was shown that EGFR is necessary for the signal support and cell transformation mediated by HER2-overexpression.

HER2/HER3 heterodimer stimulation leads to Phosphatidyl Inositol-3-Kinase (PI3K) activation acting on AKT pathway, which is responsible for mediating several oncogenic signals. The initial stimulation of tumor invasion and metastasis is mediated by activation of HER3 via PI3K pathways (Smirnova et al., 2011).

Signaling mediated by HER2/HER3-PI3K-AKT phosphorylates Tuberous Sclerosis Complex 1 and 2 (TSC1 and TSC2) and p27KIP1. As result, increased of CDK2 activity, DNA synthesis and S phase of cell cycle is observed. Thus, this process leads to protein synthesis and cell growth (Dan et al., 2002). HER2/HER3 heterodimer directly phosphorylate AKT pathways mediating caspase-9 and BAD or negatively regulate Apoptosis Signal-regulating Kinase 1 (ASK1) (Kim et al., 2001) and Forkhead Transcription Factor (FKHR) (Tang et al., 1999), stimulating tumor cell survival.

Proliferation and cell cycle progression may be stimulated by HER2/HER3 by direct activation of AKT on p21CIP1 (Zhou et al., 2001) and Murine Double Minute 2 (MDM2) (Higashiyama et al., 1997). AKT can associate with p21CIP1 and phosphorylate it at threonine residue 145 in the cell nucleus. p21CIP1 phosphorylation signal lead to the cytoplasmic localization of p21CIP1, promoting cell growth (Zhou et al., 2001). The oncoprotein MDM2 is already described as overexpressed in some tumors as displaying inhibitory function of p53 (Higashiyama et al., 1997). Also, proliferation of cell cycle progression can be facilitated through the inhibition of Glycogen Syntase Kinase 3 (GSK3), inhibiting cyclin D1. Activation of HER2/HER3 is able to promote the metabolism of carbohydrates by inhibiting GSK3. Moreover, mechanisms of invasion and metastasis are mediated by activation of $\beta$-integrin and inhibition of Matrix Metalloproteinase 2 (MMP2) (Manoukian and Woodgett, 2002). 


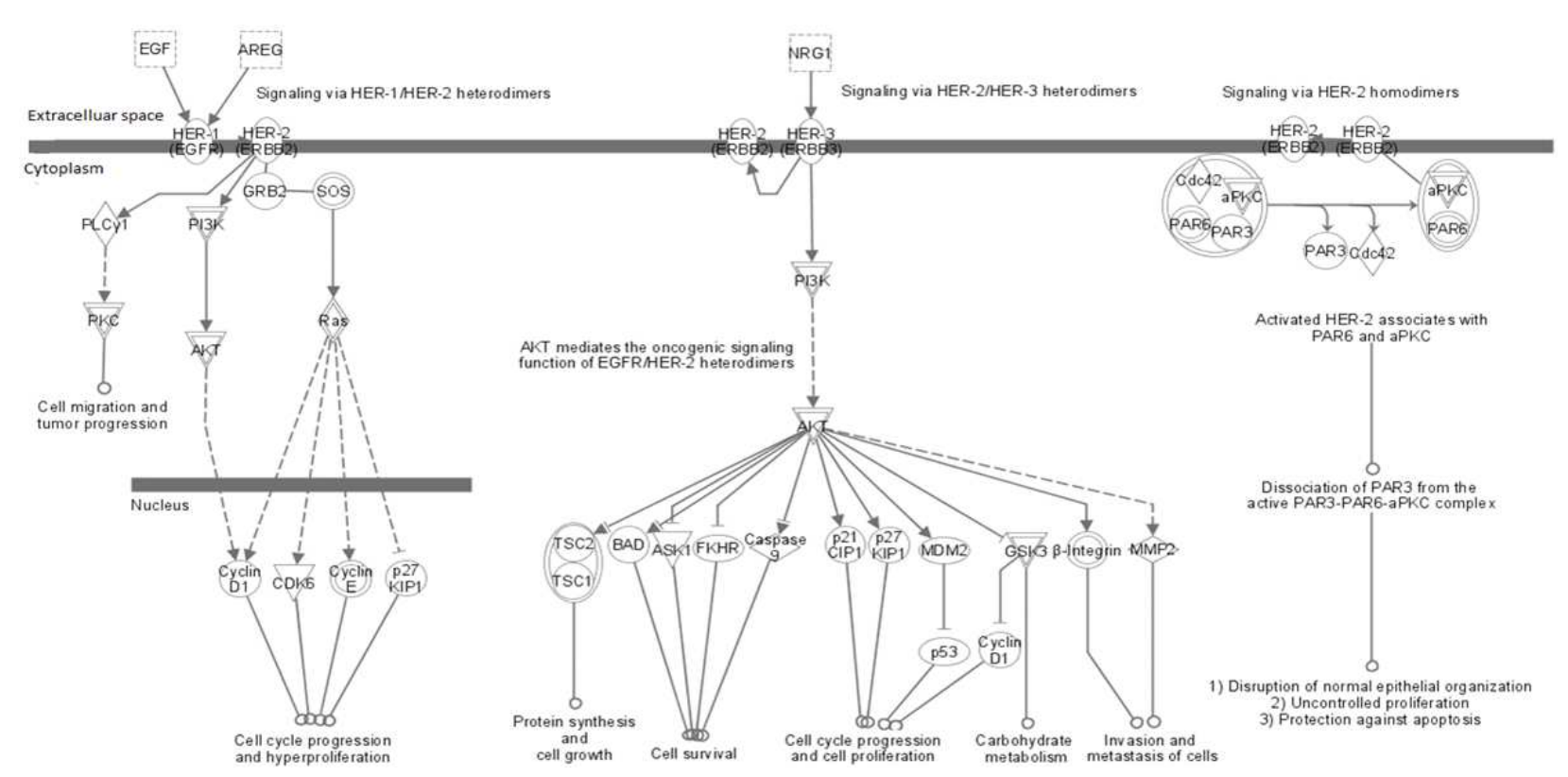

Fig. 1. Signaling pathways mediated by HER2 family members (IPA Ingenuity Systems)

HER2/HER4 heterodimer is a receptor for neuregulins and it is associated with processes of chemotaxis, cell proliferation and differentiation (Yarden and Sliwkowski, 2001).

\section{Involvement of Reactive Species in HER2 Signaling Pathways}

Reactive species are defined as organic molecules and inorganic atoms which have one or more unpaired electrons, capable of independent existence. When the unpaired electron is centered on the oxygen or nitrogen atoms they are called Reactive Oxygen Species (ROS) or Reactive Nitrogen Species (RNS), respectively. The instability of the reactive species is attributed to its configuration, which leads to a short half-life and confers high reactivity (Halliwell, 1989). In contrast, the body has an arsenal of molecules that act as antioxidant defenses scavengers of reactive species (Halliwell and Gutteridge, 2007).

The antioxidant defense system is divided into enzymatic and non-enzymatic system. The enzymatic system includes enzymes such as Superoxide Dismutase (SOD), catalase and glutathione peroxidase. The non-enzymatic system includes compounds synthesized by the body, such as glutathione, uric acid, bilirubin and sex hormones and compounds ingested in the diet, such as flavonoids, ascorbic acid and $\alpha$ tocopherol (Nijveldt et al., 2001; Schneider and Oliveira, 2004). According to Halliwell and Gutteridge (2007), the antioxidant defense can often be induced by exposing the body to reactive species and cell signaling molecules such as cytokines. Thus, oxidative stress is characterized by an imbalance between oxidant and antioxidant molecules resulting in cellular damage.

Tumor initiation may be mediated by reactive species (Cotran et al., 2010). However, it is known that resting cells remain in reduced environment, with high concentrations of antioxidants. At moderate levels of oxidative stress, the cell is stimulated to proliferate, whereas an increase in intracellular calcium and phosphorylation of proteins is involved. When levels of oxidative stress are considered higher, oxidative damage to cellular structures are installed. If such damage is not repaired and/or oxidative damage is continuous, the cell is stimulated to activate apoptosis or necrosis pathways (Halliwell, 2007). Thus, it becomes clear the regulatory role of oxidative stress in cell survival.

Studies have been shown that many products of reactive species may have a regulatory role in cancer. Mannello et al. (2007) measured levels of Malondialdehyde (MDA) and 8-epimer of prostaglandin F $2 \alpha$, two products of lipid peroxidation, in breast fluid aspirated from healthy women and breast cancer patients. It was found that levels of 8epimer of prostaglandin F2 $\alpha$ were higher in healthy women than in women with breast cancer. Also, no difference in the levels of MDA was found. Those data suggest a physiological role for 8-epimer of prostaglandin $\mathrm{F} 2 \alpha$ in normal mammary glands. Gago-Dominguez et al. (2007) propose the lipid peroxidation as a protective mechanism in breast cancer, where products of lipid peroxidation may participate in signaling cascades mediating the control of cell proliferation, differentiation and apoptosis induction. 
The involvement of reactive species, such as hydrogen peroxide, as a modulator of PI3K/AKT and p38 MAPK pathways is already reported (Angeloni et al., 2011). This pathway is activated by HER2 receptors as discussed above. In vitro studies show that anthraquinones may be able to induce apoptosis in cells that overexpress HER2 by increasing the expression of p53 and caspase 9 through mediation of reactive species (Chang et al., 2012). Seo et al. (2011) have shown that antioxidants such as genistein and quercetin are able to induce extrinsic apoptosis pathway through over-regulation of p53 and inhibits this signaling by Nuclear Factor\{kappa\} B (NF-kB). Also, as shown by Shin-Kang et al. (2011), tocotrienols reduce the activation of ERKMAPK and suppress the activation of AKT, another important pathway in HER2-overexpressed cells. Moreover, Kuo et al. (2011) demonstrated that an alkaloid can suppress the growth of tumor cells that overexpress HER2 through modulation of PI3K/AKT signaling pathway. This compound is able to interfere with the expression of cyclins D1 and cyclin E and induce apoptosis by mitochondrial pathways. Alterations in proteins of the MAPK pathway and cell surface receptors like HER2 were observed during treatment with a steroidal lactone derived from the plant Vassobia breviflora (Grogan et al., 2013). Besides, supplementation with annatto-T3 in HER2 transgenic mice showed antitumor effect related to the direct induction of oxidative stress, senescent-like growth arrest and apoptosis of tumor cells (Pierpaoli et al., 2013).

The cellular treatment inhibiting HER1/HER2 heterodimer may increase ROS leading to apoptosis. The ROS-induced apoptosis can be reversed by the presence of SOD mimic adjuvant to treatment of breast cancer (Aird et al., 2012). Also, HER2 seems to regulate Uncoupled Protein 2 (UCP2) levels and the rapid regulation of mitochondria by HER2 requires UCP2, but not its transporter activity showing the ability of HER2 to control oxidative phosphorylation (Patel et al., 2013).

\section{Therapeutics Target for HER2 and Oxidative Stress Implications}

Target strategies focusing on HER2 signaling constitutes a pivotal therapeutic in breast cancer treatment. Trastuzumab is a humanized monoclonal antibody that binds to the extracellular domain of HER2 in cancer cells and blockade the signaling transduction triggered by this pathway. Trastuzumab directly inhibits HER2 signaling by detaining its dimerization. Other effects include the activation of immune components to antibody-dependent cytotoxicity and angiogenesis inhibition (Hudis et al., 2007).

Although the clinical use of trastuzumab has brought an important advance to breast cancer therapeutic options, the use of this drug as monotherapy present some relevant toxicity. Myelosuppresion, hypersensitivity and cardiac toxicity have been reported (Genetech, 2004). The cardiac toxicity has emerged as a relevant adverse reaction that affects about $27 \%$ of patients when co-administered with anthracyclines and $13 \%$ in association with paclitaxel regimen, while trastuzumab alone had $5 \%$ of cardiotoxicity (Hudis et al., 2007; Fallah-Rad et al., 2011).

Some studies suggest that HER2 signaling repairs the anthracyclin-induced cardiac damage (Crone and Lee, 2002; Ozcelik et al., 2002). HER2 deficient mice presents a wide of characteristics related with dilated cardiomyopathy, as well the cardiomyocytes isolated from such hearts revealed increased susceptibility to anthracyclines toxicity, suggesting that HER2 signaling is a pivotal component to prevent the occurrence of heart failure (Crone and Lee, 2002).

The occurrence of oxidative stress is a mechanism of action of several chemotherapy agents. Moreover, patients submitted to chemotherapy also present systemic alterations as indicative of oxidative damage. Data from our group demonstrated that breast cancer women undergoing anthracyclines-based chemotherapy present several oxidative alterations in plasma after drug infusion (Panis et al., 2012). Interestingly, such oxidative alterations were accompanied by mild anemia development, indicating that the generation of oxidative stress (necessary to doxorubicin antitumoral effect) has a systemic toxic consequence in circulating red blood cells. We also observed other alterations as indicative of tissue damage, as augmented levels of hepatic injury enzymes and cardiac markers in breast cancer patients treated with the same protocol (unpublished data). Since the association of trastuzumab with anthracyclins seems to enhance cardiotoxicity, the mechanism underlying this side effect could involve the imbalance in oxidative stress generation. In fact, our data shows that breast cancer patients bearing tumors with HER2overexpression without previous treatment present attenuation of oxidative stress by preventing plasma lipid peroxidation and MDA formation and increasing SOD and GSH levels (Victorino et al., 2013).

An overview of trastuzumab-induced cardiac dysfunction indicates that the adjuvant use of this drug enhance the cardiac damage by a "dual-hit" mechanism. This mechanism is based on inhibition of neuregulin-1 signaling and activation of NADPH-oxidase, increasing ROS generation, since the activation of HER dimerization by neuregulin promotes mitochondrial changes by PI3/Akt signaling that decrease ROS production and promotes cell survival, apoptosis inhibition and maintenance of cardiac functions. The linkage of trastuzumab to HER2 receptor blockage is its ability to dimerize, resulting in NADPH oxidase activation and superoxide anion production. This fact can be potentiated with the co-administration of 
doxorubicin, with activation of ASK-1 and p38/jun Nterminal kinase-associated pathways, culminating in cell death by apoptosis and cardiac failure through ROS-dependent pathways (Zeglinski et al., 2011). This network seems to be cyclic and cumulative. The increased oxidative stress enhances the circulating levels of angiotensin II, a potent inhibitor or neuregulin-1, with a cumulative effect on ROS production inside cardiac cells. Furthermore, angiotensin II activates the NADPH oxidase system, leading to mitochondrial dysfunction by impairment of the electron transport chain (Nakagami et al., 2003; Cardinale et al., 2006). Trastuzumab treatment also promotes oxidative stress and apoptosis in myocardium of mice and it alters the expression of myocardial genes essential for DNA repair, cardiac and mitochondrial functions (ElZarrad et al., 2013).

Some strategies have been employed by clinicians aiming to reduce the cardiac toxicity occurrence, as the searching for pre-existing cardiac risk, early follow-up of cardiac function during trastuzumabdoxorubicin combined treatment and infusion of lesscardiotoxic doxorubicin formulations (Rayson et al., 2008). Since trastuzumab therapy indicates the need of continuous HER2 suppression for disease control (Spector and Blackwell, 2009), further studies have to be conducted aiming to understand the blockage of HER2 pathway to minimize the cardiac injury induced by oxidative stress signaling.

\section{Conclusion}

Reactive species and antioxidants mediate important cell signaling pathways that are overactive in cells with HER2 amplification/overexpression. In cancer cells, these pathways act on behalf of the tumor, leading to more aggressive characteristics such as protection from apoptosis, augment of cell survival, increase cell migration, tumor growth, proliferation and progression. Therefore, several works aim to contribute with the understanding of cellular pathways activated during tumor development and the consequences of these pathways activation in the host is not completely understood. The knowledge of the impact of HER2-overexpression in tumor cell and in host involving oxidative mechanism may contribute to the development of new specific target therapies.

\section{Author's Contributions}

All authors equally contributed in this work.

\section{Ethics}

This article is original and contains unpublished material. The corresponding author confirms that all of the other authors have read and approved the manuscript and no ethical issues involved.

\section{References}

Aird, K.M., J.L. Allensworth, I. Batinic-Haberle, H.K. Lyerly and M.W. Dewhirst et al., 2012. ErbB1/2 tyrosine kinase inhibitor mediates oxidative stress-induced apoptosis in inflammatory breast cancer cells. Breast Cancer Res Treat., 132: 10919. PMID: 21559822

Angeloni, C., E. Motori, D. Fabbri, M. Malaguti and E. Leoncini et al., 2011. H2O2 preconditioning modulates phase II enzymes through p38 MAPK and PI3K/Akt activation. Am. J. Physiol., 300: H2196-205. DOI: 10.1152/ajpheart.00934.2010

Aranda, V., T. Haire, M.E. Nolan, J.P. Calarco and A.Z. Rosenberg et al., 2006. Muthuswamy Par6-aPKC uncouples ErbB2 induced disruption of polarized epithelial organization from proliferation control. Nature Cell Biol., 8: 1235-1245. DOI: $10.1038 / \mathrm{ncb} 1485$

Barros, A.C.S.D., E.M. Barbosa, L.H. Gebrim, A. Anelli and A. Figueira Filho et al., 2001. Diagnóstico e tratamento do câncer de mama. AMB/CFM-Projeto Diretrizes.

Cardinale, D., A. Colombo, M.T. Sandri, G. Lamantia and N. Colombo et al., 2006. Prevention of highdose chemotherapy-induced cardiotoxicity in highrisk patients by angiotensin-converting enzyme inhibition. Circulation, 114: 2474-81. DOI: 10.1161/CIRCULATIONAHA.106.635144

Chang, C.Y., H.L. Chan, H.Y. Lin, T.D. Way and M.C. Kao et al., 2012. Rhein induces apoptosis in human breast cancer cells. Evidence-Based Complementary Alternative Medice, 2012: 952504-952504. DOI: 10.1155/2012/952504

Cotran, R.K., V. Kumar and T. Collins, 2010. Robbins: Patologia Estrutural e Funcional. 8th Edn., Editora Elsevier.

Crone, S.A. and K.F. Lee, 2002. Gene targeting reveals multiple essential functions of the neuregulin signaling system during development of the neuroendocrine and nervous systems. Annals N Y Acad. Sci., 971: 547-53. DOI: 10.1111/j.1749-6632.2002.tb04525.x

Dan, H.C., M. Sun, L. Yang, R.I. Feldman and X.M. Sui et al., 2002. Phosphatidylinositol 3-kinase/akt pathway regulates tuberous sclerosis tumor suppressor complex by phosphorylation of tuberin. J. Biol. Chem., 277: 35364-35370. DOI: 10.1074/jbc.M205838200

Dittmar, T., A. Husemann, Y. Schewe, J. Nofer and B. Niggemann et al., 2002. Induction of cancer cell migration by epidermal growth factor is initiated by specific phosphorylation of tyrosine 1248 of cerbB-2 receptor via EGFR1. FASEB J. DOI:10.1096/fj.02-0096fje 
Dogan, I., A. Cumaoglu, A. Aricioglu and A. Ekmekci, 2011. Inhibition of ErbB2 by Herceptin reduces viability and survival, induces apoptosis and oxidative stress in Calu-3 cell line. Molecular Cellular Biochem., 347: 41-51. DOI: 10.1007/s11010-010-0610-7

ElZarrad, M.K., P. Mukhopadhyay, N. Mohan, E. Hao and M. Dokmanovic et al., 2013. Trastuzumab alters the expression of genes essential for cardiac function and induces ultrastructural changes of cardiomyocytes in Mice. PLoS ONE, 8: e79543e79543. DOI: 10.1371/journal.pone.0079543

Fallah-Rad, N., J.R. Walker, A. Wassef, M. Lytwyn and S. Bohonis et al., 2011. The utility of cardiac biomarkers, tissue velocity and strain imaging and cardiac magnetic resonance imaging in predicting early left ventricular dysfunction in patients with human epidermal growth factor receptor IIpositive breast cancer treated with adjuvant trastuzumab therapy. J. Am. Coll. Cardiol., 57: 2263-70. DOI: 10.1016/j.jacc.2010.11.063

Gago-Dominguez, M., X. Jiang and J.E. Castelao, 2007. Lipid peroxidation, oxidative stress genes and dietary factors in breast cancer protection: A hypothesis. Breast Cancer Res., 9: 201-201. DOI: 10.1186/bcr1628

Genetech, 2004. Herceptin (trastuzumab): Full prescribing information. South San Francisco, CA: Genentech.

Graus-Porta, D., R.R. Beerli, J.M. Daly and N.E. Hynes, 1997. ErbB-2, the preferred heterodimerization partner of all ErbB receptors, is a mediator of lateral signaling. EMBO J., 16:1647-1655. DOI: 10.1093/emboj/16.7.1647

Grogan, P.T., K.D. Sleder, A.K. Samadi, H. Zhang and B.N. Timmermann et al., 2013. Cytotoxicity of withaferin a in glioblastomas involves induction of an oxidative stress-mediated heat shockresponse while altering Akt/mTOR and MAPK signaling pathways. Invest New Drugs, 31: 545-57. DOI: $10.1007 / \mathrm{s} 10637-012-9888-5$

Gutierrez, C. and R. Schiff, 2011. HER2 biology, detection and clinical implications. Arch. Pathol. Laboratory Medice, 135: 55-62. DOI: 10.1043/2010-0454-RAR.1

Halliwell, B. and J.M.C. Gutteridge, 2007. Free Radicals in Biology and Medicine. In: Oxygen is a Toxic Gas: An Introduction to Oxygen Toxicity and Reactive Species, Oxford University, New York, pp: 1-28.

Halliwell, B., 1989. Tell me about free radicals, doctor: A review. J. Royal Society Med., 82: 747-752.

Halliwell, B., 2007. Oxidative stress and cancer: Have we moved forward? Biochemical J., 401: 1-11. DOI: $10.1042 / \mathrm{BJ} 20061131$

Higashiyama, M., O. Doi, K. Kodama, H. Yokouchi and T. Kasugai et al., 1997. MDM2 gene amplification and expression in non small cell lung cancer: Immunohistochemical expression of its protein is a favourable prognostic marker in patients without $\mathrm{p} 53$ protein accumulation. British J. Cancer, 9: 1302-1308.
Hudis, C.A., W.E. Barlow, J.P. Costantino, R.J. Gray and K.I. Pritchard et al., 2007. Proposal for standardized definitions for efficacy end points in adjuvant breast cancer trials: The STEEP system. J. Clin. Oncol., 25: 2127-32. DOI:10.1200/JCO.2006.10.3523

Hynes, N.E. and H.A. Lane, 2005. Erbb receptors and cancer: The complexity of targeted inhibitors. Nat. Rev. Cancer, 5: 341-353. DOI: 10.1038/nrc1609

INC, 2004. Controle do câncer de mama: Documento de consenso. Ministério da Saúde. Instituto Nacional do Câncer.

Janes, P.W., R.J. Daly, A. De Fazio and R.L. Sutherland, 1994. Activation of the rassignalling pathway in human breast cancer cells overexpressing erbB-2. Oncogene, 9: 3601-8.

Johnson-Thompson, M.C. and J. Guthrie, 2000. Ongoing research to identify environmental risk factors in breast carcinoma. Cancer, 88: 1224-9.

Kim, A.H., G. Khursigara, X. Sun, T.F. Franke and M.V. Chao, 2001. Akt phosphorylates and negatively regulates apoptosis signal-regulating kinase. Molecular Cellular Biol., 21: 893-901. DOI: 10.1128/MCB.21.3.893-901

Kuo, H.P., T.C. Chuang, M.H. Yeh, S.C. Hsu and T.D. Way et al., 2011. Growth suppression of HER2-overexpressing breast cancer cells by berberine via modulation of the HER2/PI3K/Akt signaling pathway. J. Agric. Food Chem., 59: 8216-24. DOI: $10.1021 /$ jf2012584

Kurebayashi, J., 2001. Biological and clinical significance of HER2 overexpression in breast cancer. Breast Cancer, 8: 45-51.

Mannello, F., G.A. Tonti, S. Pagliarani, S. Benedetti and F. Canestrari et al., 2007. The 8-epimer of prostaglandin F2a, a marker of lipid peroxidation and oxidative stress, is decreased in the nipple aspirate fluid of women with breast cancer. Int. J. Cancer, 120: 1971-6. DOI: 10.1002/ijc.22522

Manoukian, A.S. and J.R. Woodgett, 2002. Role of glycogen synthase kinase-3 in cancer: regulation by Wnts and other signaling pathways. Adv. Cancer Res., 84: 203-29.

Nahta, R., D. Yu, M.C. Hung, G.N. Hortobagyi and F.J. Esteva, 2006. Mechanisms of disease: Understanding resistance to HER2-targeted therapy in human breast cancer. Nat. Clin. Pract. Oncol., 3 269-280. DOI: 10.1038/ncponc0509

Nakagami, H., M. Takemoto and J.K. Liao, 2003. NADPH oxidase-derived superoxide anion mediates angiotensin II-induced cardiac hypertrophy. J. Molecular Cellular Cardiol., 35: 851-9. DOI: 10.1016/S0022-2828(03)00145-7

Nijveldt, R.J., E. van Nood, D.E. van Hoorn, P.G. Boelens and K. van Norren et al., 2001. Flavonoids: A review of probable mechanisms of action and potencial applications. Am. J. Clin. Nutrit., 74: 418-425. 
Ozcelik, C., B. Erdmann, B. Pilz, N. Wettschureck and S. Britsch et al., 2002. Conditional mutation of the ErbB2 (HER2) receptor in cardiomyocytes leads to dilated cardiomyopathy. Proc. Nat. Acad. Sci. USA, 99: 8880-5. DOI: 10.1073/pnas. 122249299

Panis, C., A.C. Herrera, V.J. Victorino, F.C. Campos and L.F. Freitas et al., 2012. Oxidative stress and hematological profiles of advanced breast cancer patients subjected to paclitaxel or doxorubicin chemotherapy. Breast Cancer Res. Treatment, 133: 89-97. DOI: 10.1007/s10549-011-1693-x

Patel, N., A. Barrientos and R. Landgraf, 2013. The growth factor receptor ERBB2 regulates mitochondrial activity on a signaling time scale. J. Biol. Chem., 288: 3525365. DOI: 10.1074/jbc.M113.478271

Perou, C.M., T. Sorlie, M.B. Eisen, M. van de Rijn and S.S. Jeffrey et al., 2000. Molecular portraits of human breast tumours. Nature, 406: 747-752. DOI: $10.1038 / 35021093$

Pierpaoli, E., V. Viola, A. Barucca, F. Orlando and F. Galli et al., 2013. Effect of annatto-tocotrienols supplementation on the development of mammary tumors in HER-2/neu transgenicmice. Carcinogenesis, 34: 1352-60. DOI: 10.1093/carcin/bgt064

Rayson, D., D. Richel, S. Chia, C. Jackisch and S. van der Vegt et al., 2008. Anthracycline-trastuzumab regimens for HER2/neu-overexpressing breast cancer: current experience and future strategies. Annals Oncol., 19:1530-9. DOI: 10.1093/annonc/mdn292

Schneider, C.D. and A.R. Oliveira, 2004. Radicais livres de oxigênio e exercício: Mecanismos de formação e adaptação ao treinamento físico. Rev. Brasil. Med., 10: 4-4.

Seo, H.S., H.S. Choi, H.S. Choi, Y.K. Choi and J.Y. Um et al., 2011. Phytoestrogens Induce apoptosis via extrinsic pathway, inhibiting nuclear factor\{kappa\}B signaling in HER2-overexpressing breast cancer cells. Anticancer Res., 31: 3301-13.

Shin-Kang, S., V.P. Ramsauer, J. Lightner, K. Chakraborty and W. Stone et al., 2011. Tocotrienols inhibit AKT and ERK activation and suppress pancreatic cancer cell proliferation by suppressing the ErbB2 pathway. Free Radical Biol. Med., 51: 116474. DOI: $10.1016 /$ j. freeradbiomed.2011.06.008

Sioshansi, S., K.E. Huber and D.E. Wazer, 2011. The implications of breast cancer molecular phenotype for radiation oncology. Frontiers Oncol., 28: 12-12. DOI: $10.3389 /$ fonc. 2011.00012

Smirnova, T., Z.N. Zhou, R.J. Flinn, J. Wyckoff and P.J. Boimel et al., 2011. Phosphoinositide 3-kinase signaling is critical for ErbB3-driven breast cancer cell motility and metastasis. Oncogene, 31: 706-15. DOI: $10.1038 /$ onc.2011.275
Spector, N.L. and K.L. Blackwell, 2009. Understanding the mechanisms behind trastuzumab therapy for human epidermal growth factor receptor 2positive breast cancer. J. Clin. Oncol., 27: 583847. DOI: $10.1200 /$ JCO.2009.22.1507

Tang, E.D., G. Nunez, F.G. Barri and K.L. Guan, 1999. Negative regulation of the forkhead transcription factor FKHR by Akt. J. Biol. Chem., 274: 16741-6. DOI: $10.1074 /$ jbc.274.24.16741

Tanizaki, J., I. Okamoto, S. Fumita, W. Okamoto and K. Nishio et al., 2011. Roles of BIM induction and survivin downregulation in lapatinib-induced apoptosis in breast cancer cells with HER2 amplification. Oncogene, 30: 4097-106. DOI: 10.1038/onc.2011.111

Victorino, V.J., F.C. Campos, A.C. Herrera, A.N. Colado Simão and A.L. Cecchini et al., 2013. Overexpression of HER-2/neu protein attenuates the oxidative systemic profile in women diagnosed with breast cancer. Tumour Biol.

Vieira, D.S.C., R.M. Dufloth, F.C.L. Schmitt and L.C. Zeferino, 2008. Carcinoma de mama: novos conceitos na classificação. Breastcancer: New concepts in classification. Rev. Brasil. Ginecol. Obstetrícia, 30: 42-7. DOI: 10.1590/S0100-72032008000100008

WHO, 2014. Health topics: Cancer. World Health Organization.

Yarden, Y. and M.X. Sliwkowski, 2001. Untangling the Erbb signalling network. Nat. Rev. Molecular Cell Biol., 2: 127-137. DOI: 10.1038/35052073

Yarden, Y., 2001. Biology of HER2 and its importance in breast cancer. Oncology, 61: 1-13. DOI: $10.1159 / 000055396$

Zeglinski, M., A. Ludke, D.S. Jassal and P.K. Singal, 2011. Trastuzumab-induced cardiac dysfunction: A 'dual-hit'. Exp. Clin. Cardiol., 16: 70-4.

Zhou, B.P., Y. Liao, W. Xia, B. Spohn and M.H. Lee et al., 2001. Cytoplasmic localization of p21Cip1/WAF1 by Akt-induced phosphorylation in HER-2/neu overexpressing cells. Nat. Cell Biol., 3: 245-252. DOI: $10.1038 / 35060032$

Zhou, X. and Y.M. Agazie, 2011. The signaling and transformation potency of the overexpressed HER2 protein is dependent on the normallyexpressed EGFR. Cellular Signal., 24: 140-50. DOI: $10.1016 /$ j.cellsig.2011.08.015 